recrutement des infirmières pour les hôpitaux belges, et organisa des cours à cet effet. Enfin son service d'information a pu répondre à plus de 25,000 demandes, et a fait parvenir des milliers d'exemplaires d'un colis type de $8 \mathrm{fr}$. aux soldats belges nécessiteux.

\title{
BULGARIE
}

\section{Eléonore, reine des Bulgares, protectrice de la Croix-Rouge bulgare}

\section{(Nécrologie)}

De même que nous avions, à l'occasion de sa fin tragique et douloureuse, consacré, en 1908, quelques lignes à l'impératrice d'Autriche, protectrice de la Croix-Rouge autrichienne, de même nous sommes heureux de pouvoir insérer sur la reine Eléonore de Bulgarie, récemment décédée, la note suivante, que nous devons à l'obligeance de MM. Guéchoff, président de la Société bulgare de la Croix-Rouge et Mikoff, délégué de la Croix-Rouge bulgare à Genève :

«S. M. la reine Eléonore, fille du prince llenri IV de Reuss-Köstritz, née le 22 août $1860^{1}$, à Trepchen près Tzwilickau, était la seconde épouse de S. M. Ferdinand Ier, roi des Bulgares. Leur mariage fut célébré le 17 l'évrier 1908, à Schloss Osterstein, près Géra. La grande duchesse Marie Pavlovna, épouse du grand duc Vladimir Alexandrovitch, qui prit part à la guerre russo-turque de 1877-78, était sa cousine germaine.

"Les sentiments de profonde humanité qui distinguaient la défunte lui ont valu la gratitude émue des populations, et son nom était toujours prononcé avec une vénération reconnaissante. Dans le château de son frère, le prince Henri

14 ans jour pour jour avant la signature de la Convention de Genève. 
$\mathrm{XIV}$, près de Vienne où sa vie s'écoulait, la reine Eléonore s'était consacrée tout entière aux cuvres d'assistance et cle charité. Les sours de l'hôpital de Sainte Anne dans la capitale autrichienne racontent que tous les ans elle avait l'habitude de recueillir chez elle des enfants convalescents, au rétablissement desquels elle vouait les soins d'une vraie mère.

"Pendant la guerre russo-japonaise (1904-190万), elle se fit scour de charité, et se mit à la tête du personnel du deuxième convoi sanitaire envoyé en Mandchourie aux frais de la grande duchesse Marie Pavlovna ${ }^{2}$. Dans des circonstances des plus difficiles elle s'est acquittée de sa mission avec beaucoup de tact et de dévouement, ce qui lui valut l'admiration et l'estime universelles. Témoin oculaire des horreurs de la guerre, elle tendit tous ses efforts vers l'organisation du service de secours aux soldats blessés ou malades. Pendant tout l'hiver elle travailla dans un train sanitaire sur la ligne de l'Asie Orientale faisant le service entre Vladivostok, Charbin et Irkoutsk. Dans les campagnes mandchouriennes elle a bravé tous les périls.

"La reine Eléonore vint en Bulgarie le 8 mars 1908. Un accueil enthousiaste lui fut fait par la nation entière. Sn première résidence fut le château d'Euxinograde, au nord. de Varna, sur cette côte de la mer Noire qui, par la beauté de ses sites, évoque le souvenir de la Riviera. Dès les premiers jours, la reine Eléonore se mit à étudier les différentes institutions d'assistance et de charité du pays, prit à tâche d'en créer de nouvelles et de contribuer à leur prospérité. Toutes les fois qu'il était question d'élever un bâtiment affecté à une couvre de charité, elle faisait soumettre les plans à son approbation et s'intéressait activement au choix de l'emplacement. En peu de temps la reine Eléonore visita tous les sanatoriums, tous les établissements thérapeutiques, toutes les stations climatériques et thermales. A l'occasion des rêtes de Pâques, de Noël, du jour de l'an

1 Voy. notre article sur les soeurs de charité pendant la guerre russo-japonaise, T. XXXIX, 1908, p. 240. 
ou d'une fête politique quelconque, elle faisait des dons considérables en argent et en nature, pour subvenir aux besoins des pauvres, des souffrants et des infirmes. Si quelque fléau national, quelque calamité sociale survenait, tremblement de terre, inondation, etc., aussitôt elle était sur les lieux pour mieux se rendre compte des secours à apporter aux victimes.

" En 1910, la reine Eléonore prend l'initiative d'une association ayant pour but d'entretenir un courant de charité et de compassion dans le public, de préparer les femmes de bonne volonté pour le service de sœurs de charité et de venir en aide en temps de guerre à la Croix-Rouge. Sur sa proposition une société est fondée "La Samaritaine", par les soins de laquelle plus tard, en 1911, des cours théoriques et pratiques de secours aux blessés sont organisés. La reine Eléonore honora de sa présence presque toutes les leçons, ainsi que les examens de cette institution. Les diplômes de fin d'études ont été distribués personnellement par elle aux premières "Samaritaines" reçues.

"Pendant la première guerre balkanique (1912), par ses visites assidues aux différents hôpitaux et infirmeries de campagne, aux stations de ravitaillement et au front même, elle était devenue l'ange-gardien et la consolatrice des souffrants, tandis que par ses soins vraiment maternels à l'égard des officiers et soldats blessés ou malades elle avait gagné tous les cœurs ${ }^{1}$. Elle devint la plus populaire des femmes pendant la guerre. Portant le costume de sœur de charité, elle prêchait d'exemple. Elle pansait elle-même les héros, elle leur administrait les médicaments, assistait au passage des trains sanitaires ou entreprenait des tournées d'inspection dans les régions où sévissait le choléra. Sur sa cassette particulière étaient prélevés les frais d'entretien de deux sanatoriums, où travaillaient des médecins et des spécialistes étrangers appelés à cet effet.

"Pendant la seconde guerre balkanique (1913) ", elle se

1 Nous avons mentionné en son temps le beau rôle joué par la reine de Bulgarie. Voy. T. XLIV, 1913, pp. 20, 33, 155.

2 Voy. T. XIN, 1914, p. 111. 
rendit à Kustendil, en compagnie du chef du service sanitaire de l'armée en campagne, prodigua ses soins aux scldats blessés et malades du lycée de garçons, transformé en hôpital, visita le lycée de jeunes filles, aménagé également en hôpital d'une centaine de lits et entretenu à ses frais personnels. Il faut noter qu'en ce temps-là, Kustendil était tout à fait à proximité du front et que là affluaient le plus grand nombre de soldats ayant besoin de secours médicaux. De retour à Sofia, elle prit les mesures nécessaires pour que les centaines d'hommes et de femmes, réfugiés de Macédoine et blessés par les projectiles ennemis, fussent admis d'urgence tans les hôpitaux. Elle s'occupa de l'installation des 130,000 fugitifs de cette province, qui avaient quitté leurs foyers pour venir chercher le salut en Bulgarie. Elle dépensa des sommes énormes pour l'entretien de ces infortunés, conlamnés sans cela à mourir de faim et de froid.

"Au commencement de 1914, la reine Eléonore entreprit un long voyage à l'étranger. Elle se rendit tout d'abord à Vienne, dans le but d'étudier plus à fond l'organisation des hôpitaux dans cette ville et de pouvoir mieux perfectionner certaines branches du service militaire en Bulgarie. C'est ainsi qu'elle entama des pourparlers avec une maison viennoise, en vue d'une fourniture de bras et de jambes artificiels pour les invalides. Sur son initiative, des cours de couture et le tricotage furent institués à Sofia, en faveur de ces malheureux, et à la fin de ces cours elle gratifia chacun des participants d'une machine à coudre ou à tricoter. Elle s'apprêtait à partir pour l'Amérique, afin de visiter les principales cliniques et institutions sanitaires des Etats-Unis, et de faire venir en Bulgarie des sœurs de charité spécialisées dans les différents services ${ }^{1}$, mais dut remettre son voyage d̀ des jours meilleurs.

"En septembre 1915, lorsque la Bulgarie intervint dans la. grande guerre aux côtés des Puissances centrales, la reine Eléonore eut avec le commandant en chef de l'armée en campagne, le général Jékoff, une entrevue dont l'objet

1 Voy. T. XLV, 1914, p. 60. 
fut l'étude de la question de l'hospitalisation des blessés. Dés le début des hostilités, accompagnée de MM. les Drs Rousseff, Zolotovitz et Goldamer, elle partit pour Kustendil. où avaient été installés les premiers officiers et soldats blessés. Sur son ordre la plupart furent évacués sur Sofia. De Kustendil elle alla à Guéchévo et de là revint à Radomir. pour organiser dans ces deux localités, comme elle l'avait fait à Kustendil, des stations de ravitaillement pour blessés.

"Elle visitait souvent les hôpitaux, s'entretenait avec les blessés et malades sans distinction de nationalité, adressait à chacun quelques paroles de réconfort et de consolation, et les gratifiait de cadeaux en argent, en objets, en conserves, etc. Elle n'oublia pas les prisonniers de guerre envers lesquels elle fit preuve d'une sollicitude maternelle. Elle se rendit à Plovdiv, Stara-Zagora, Gorno-Panitchérévo, où sont internés des milliers de Serbes, et leur fit distribuer du linge et des couvertures chaudes. Accompagnée par M. le Dr.Goldamer, elle inspecta les principaux hôpitaux où se trouvaient cles soldats amputés, fit transférer ces derniers à Sofia et les mit à même de profiter de l'institut orthopédique qui venait d'être installé dans la capitale.

* Conjointement avec le roi elle était la protectrice de la Société bulgare de la Croix-Rouge, laquelle appréciait hautement sa compétence et ne manquait jamais de prendre en considération ses conseils dans toutes ses entreprises.

"En outre dix sociétés, formant "l'Union des Sociétés de bienfaisance pendant la guerre " - laquelle déploie une activité considérable dans le domaine de la charité -, proclamèrent la reine leur protectrice.

"Physiquement ébranlée et exténuée par les soucis et par son labeur constant en faveur des blessés, la reine Eléonore se vit obligée d'aller se reposer et se faire soigner à l'étranger. Mème de loin elle ne cessa de s'intéresser au sort des malades et des malheureux, sachant traduire en actes sa compassion et leur faisant généreusement envoyer des lons par l'intermédiaire des différentes institutions de bienfaisance. A son anniversaire de naissance, le 22 août 1916 , 


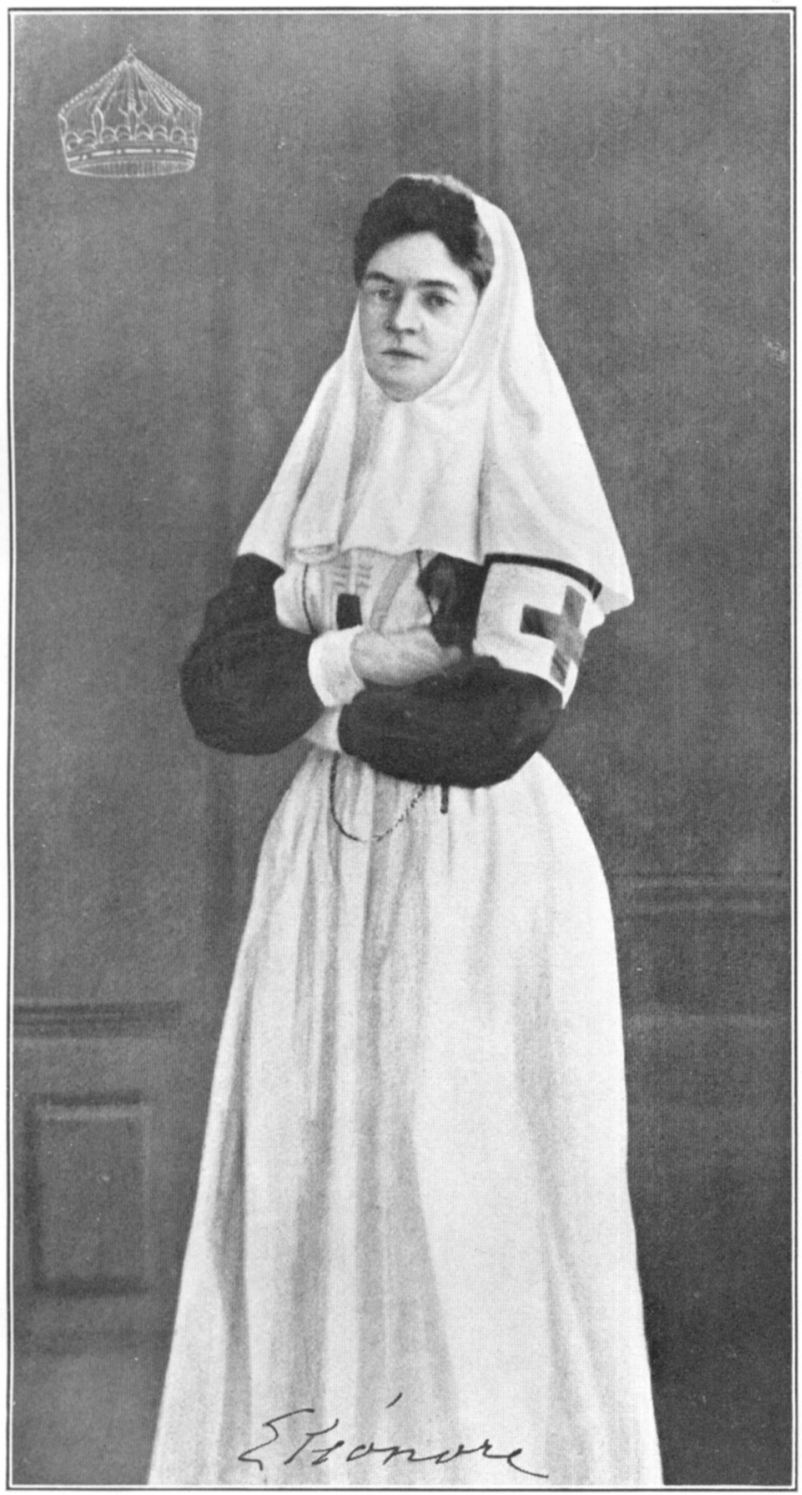


se trouvant en Saxe, dans la station climatérique du Weisse Hirsch, près de Dresde, elle ordonna de doubler, à ses frais, la ration de tous les officiers et soldats malades ou blessés soignés dans les divers établissements médicaux. Elle envoya des cadeaux au régiment $\mathrm{N}^{\circ} 24$ de la Mer Noire, dont elle était le chef, ainsi qu'à d'autres régiments qui avaient de dures campagnes à leur actif et s'étaient distingués par leurs faits d'armes. En outre elle fit inviter, à une table dressée spécialement à leur intention, les 300 soldats blessés du sanatorium de $M$. le Dr Lahmann. Elle vécut pendant quelques mois là, puis eut à subir une grave opération, et plus tard à Halle, une seconde, plus grave encore, dans le sanatorium de $\mathrm{M}$. le $\mathrm{D}^{\mathrm{r}}$ Schmieden. Tout d'abord elle en ressentit un soulagement relatif, mais dans la suite son mal s'accentua et sa faiblesse devint grande. Après un traitement de près d'un an, elle rentra à Sofia qu'elle quitta. bientôt pour aller respirer l'air bienfaisant du littoral à Euxinograde. Là, elle se confia aux soins de médecins bulgares et de deux sœurs de charité.

"Durant sa maladie, la reine Eléonore disait souvent : "Je ne me sens pas si mal: si je pouvais seulement me nourrir pour avoir la force de continuer l'œuvre que j'ai encore à accomplir!" L'une des deux scurs de charité raconte qu'elle ne se plaignait pas, mais que sa pensée allait aux malades, aux orphelins, aux souffrants. Un peu amaigrie, modeste et pensive, elle se tenait à la fenêtre, le regard perdu dans l'horizon lointain de la Mer Noire. Elle soupirait et se tourmentait à la pensée qu'elle ne pouvait plus se vouer à ses cuvres de charité. En 1917, elle passa l'anniversaire de son jour de naissance à Euxinograde sur son lit de maladie. C'est là qu'elle lut les nombreuses dépêches de félicitations qui lui furent adressées à cette occasion. Sa santé extrêmement chancelante ne l'empêcha pas de marquer son jour de naissance d'une nouvelle initiative humanitaire : elle commanda dans les chantiers de la flotte, à Varna, un canot commémoratif, à garnir de clous au profit des blessés pauvres et leurs familles. Elle donna sa photographie signée de sa propre main, ainsi que d'autres objets, pour être ven- 
dus aux enchères dans un but de bienfaisance. Rien que pendant le premier jour, à Varna, le produit du "cloutage " du canot commémoratif se monta à $25,000 \mathrm{fr}$., tandis que sa photographie fut vendue $2,000 \mathrm{fr}$. Civils et militaires rivalisaient de générosité.

"Dans les premiers jours de septembre 1917, la santé de la reine Eléonore empira. Le 11 septembre, un grand affaiblissement du cour survint, mais elle put recevoir toute la famille royale. Le 12 septembre, elle remercia en termes touchants le roi, les princes et les princesses de l'attention particulière qu'ils lui avaient témoignée et de leur grande sollicitude pour elle. Peu après, elle entrait en agonie; le même jour elle expirait, laissant dans les pleurs et la douleur la famille royale et tout le peuple bulgare. La reine Eléonore est morte à l'âge de 57 ans.

"Sa dépouille mortelle, embaumée dans un beau sarcophage, fut transportée d'Euxinograde à Sofia. Le service de requiem fut célébré d'après le rite protestant, devant une affluence de plusieurs milliers d'assistants. Pendant l'exposition du corps dans l'église luthérienne allemande, ce qui impressionnait, c'était le défilé incessant de blessés et d'invalides, qui rendaient un dernier hommage de pieuse reconnaissance à leur grande bienfaitrice. Ce qui frappait aussi, c'était le fait que parmi les nombreuses couronnes, il y en avait une qui était envoyée par les officiers et soldats anglais, prisonniers de guerre en Bulgarie. Le Comité central de la Société bulgare de la Croix-Rouge, les chefs des missions sanitaires des Croix-Rouges étrangères, toutes les sœurs de charité et les samaritaines de toutes nationalités, des délégations des différentes sociétés de bienfaisance, etc., prirent part aux funérailles, qui eurent lieu le 18 septembre 1917.

"Suivant le vou exprimé par elle, son corps fut inhumé à Boiana, village situé aux pieds de la montagne, près de Sofia, dans la cour de l'église historique de Saint-Pantéleimon, antique sépulture royale.

"La reine Eléonore était la bonté même et l'incarnation de la charité. En sa personne, ce n'est pas la Bulgarie seule, c'est l'Europe entière qui perd une des plus zélées protago- 
nistes de l'idée internationale de l'amour envers le prochain, l'idée mème dont l'cuvre sublime de la Croix-Rouge, appuyée sur la Convention de Genève, est la réalisation pratique."

\section{CUBA}

\section{Nouveau changement dans la présidence de la Société cubaine}

La présidence de la Société nationale cubaine, qui était exercée par M. le colonel José Marti, ainsi que nous l'annonçait, une lettre de La Havane du 30 mai ${ }^{1}$, a passé de nouveau ell d'autres mains, ainsi qu'il résulte de la communication suivante :

La Havane, le 12 septembre 1917.

"Monsieur le Président du Comité International de la Croix-Rouge.

«Monsieur le Président,

"Nous avons l'honneur de porter à votre connaissance que notre Président, M. le colonel José Marti y Zayas Bazan, a été désigné par S. Ex. M. le Major Général Mario (i. Menocal, IIonorable Président de la République de Cuba, pour occuper le ministère de la Guerre.

"Monsieur le Président de la République a sanctionné par décret No 1283 , du 4 août 1917, et d'accord avec la décision de notre Assemblée Suprême, la nomination de $M$. le colonel Miguel Varona $y$ del Castillo, comme successeur de M. le colonel José Marti y Zayas Bazan, aux fonctions de délégué officiel du Gouvernement, Président. de l'Assemblée Suprême et du Comité Exécutif de notre Société.

"Veuillez agréer, Monsieur le Président, les assurances de 16s respectueux hommages.

"Pour le Comité Exécutif.

"Le Secrétaire général:

(I) Prof. E. Sanchez de Fuentes y Pelaez."

1 Voy. T. XLVIII, 1917, p. 421. 